\title{
Field-like spin-orbit torque induced by bulk Rashba channels in GeTe/NiFe bilayers
}

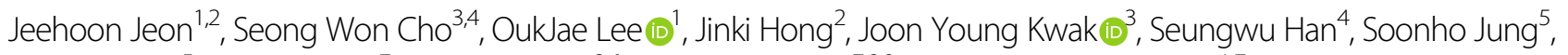

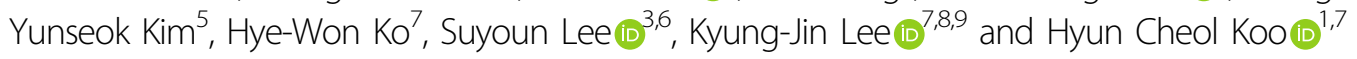

\begin{abstract}
Most studies of the Rashba effect have focused on interfacial Rashba spin-orbit coupling. Recently, bulk Rashba materials have attracted considerable interest owing to their potential to enhance the Rashba spin-orbit torque. By employing a bulk Rashba material, GeTe, as a spin-orbit channel in GeTe/NiFe bilayers, a large field-like spin-orbit torque up to $15.8 \mathrm{mT} /\left(10^{7} \mathrm{~A} \mathrm{~cm}^{-2}\right)$ is measured. This value is one of the largest reported field-like torques and is attributed to the interfacial spin-orbit coupling being enhanced by the bulk Rashba effect in the GeTe channel. Furthermore, the large field-like torque is maintained even for a $20-n m$-thick NiFe layer. This unconventional dependence on the thickness of both the GeTe and NiFe layers cannot be described by conventional theory, but it is believed to stem from the additional bulk Rashba effect-induced term. The large field-like torque over a wide range of ferromagnet thicknesses results in scalable in-plane spin-orbit torque devices. This result calls for a further theoretical study on spin transport in heterostructures, including bulk Rashba materials.
\end{abstract}

\section{Introduction}

Rashba spin-orbit coupling (RSOC) is a crucial element for spin-based information devices. The control of spin precession concomitant with the modulation of RSOC by an electric field has been theoretically suggested and experimentally demonstrated using a two-dimensional quantum well $^{1-6}$. In addition to gate-controlled spin precession, the RSOC also contributes to spin-orbit torques (SOTs) in a normal metal (NM)/ferromagnet (FM) bilayer, the basic structure for SOT-active devices, where inversion symmetry breaks at the NM/FM interface gives rise to $\mathrm{RSOC}^{6-10}$. As the SOT serves as a writing scheme for energy-efficient spintronic devices ${ }^{11-13}$, enhancing the RSOC effect in NM/ FM bilayers is an important task for practical applications.

Most RSOC studies have focused on interfacial effects in two-dimensional systems or bilayers. Considering

Correspondence: Suyoun Lee (slee_eels@kist.re.kr) or

Kyung-Jin Lee (kjlee@kaist.ac.kr) or Hyun Cheol Koo (hckoo@kist.re.kr)

${ }^{1}$ Center for Spintronics, Korea Institute of Science and Technology, Seoul, Korea

${ }^{2}$ Department of Applied Physics, Korea University, Sejong, Korea

Full list of author information is available at the end of the article

These authors contributed equally: Jeehoon Jeon, Seong Won Cho spin transport, it is expected that the RSOC effect becomes stronger when the RSOC is present in the bulk of a system rather than being limited to just the interface. Recent studies have revealed that several polar crystals, such as $\mathrm{BiTeCl}^{14}, \mathrm{BiTeI}^{15}$, and $\mathrm{GeTe}^{16}$, have strong bulk RSOC, where the bulk inversion asymmetry is related to the ferroelectricity. These materials are classified as ferroelectric-Rashba semiconductors ${ }^{17,18}$. Among these materials, $\alpha-G e T e$ is remarkable in that it has a relatively high ferroelectric Curie temperature of $\sim 670 \mathrm{~K}^{19}$, and its ferroelectricity survives at a thickness of $\sim 5 \mathrm{~nm}^{20}$. In addition, a spin- and angle-resolved photoemission spectroscopy (SARPES) study reported that GeTe has a large Rashba constant of $\sim 4.2 \times 10^{-10}$ $\mathrm{eV} \mathrm{m}^{21}$.

The SOT strength in NM/FM bilayers is related to the spin Hall conductivity of the spin-orbit channel and NM layer. The aforementioned attractive features of GeTe have led to recent density functional theory calculations of the spin Hall conductivity of GeTe and its controllability through the modulation of electric polarization ${ }^{22,23}$. Even considering various values of electric polarizations, 
however, these theoretical studies predicted that the spin Hall effect (or spin Hall angle) of bulk Rashba GeTe at the Fermi level is not superior to that of Pt, a widely used spin-orbit channel for SOT devices. This result is attributed to the fact that the SOC effect of GeTe is mainly observed in the valence bands (Te $5 p$-orbitals) below the Fermi level due to Ge vacancies.

Despite the above prediction, it is worthwhile to experimentally investigate the SOT in GeTe/FM bilayers because the above theories ${ }^{24,25}$ considered only bulk GeTe. In GeTe/ FM bilayers, there is an additional inversion asymmetry at the GeTe/FM interface. Recent studies for NM/FM bilayers with centrosymmetric NM materials have suggested that the NM/FM interface can substantially contribute to the $\mathrm{SOT}^{9,10}$. In this respect, an important question has remained unexplored: what happens to the SOT of NM/FM bilayers when the NM layer is a bulk Rashba material? In this work, we address this question by investigating the SOTs in GeTe/ $\mathrm{Ni}_{81} \mathrm{Fe}_{19}$ bilayers using the second-harmonic Hall voltage measurement ${ }^{24}$, from which we extract SOT magnitudes.

\section{Results}

Polar crystal structure of a-GeTe with a strong bulk Rashba effect

The X-ray diffraction (XRD) $2 \theta$ scan of a single-layer GeTe film [Fig. 1a] shows that the film is oriented with its hexagonal $(000 h)$ axis normal to the $\mathrm{Si}(111)$ substrate. The full width at half maximum (FWHM) of the GeTe (0003) reflection is estimated to be $\sim 0.075^{\circ}$ [Supplementary Fig. S1a], indicating the high crystalline quality of the film. In Fig. 1b, the XRD $\phi$-scan of the GeTe(0-114) shows that it has threefold rotational symmetry mixed with a small amount of twinned grains, which is consistent with the rhombohedral crystal structure of $\alpha$ GeTe. The XRD reciprocal lattice map of the GeTe film indicates that the film is fully relaxed from the substrate or free of stress [Supplementary Fig. S1b].

Figure 1c shows a fast Fourier transform (FFT)-filtered atomic-resolution scanning transmission electron microscopy (STEM) image, which clearly indicates Te (brighter balls) and Ge (dimmer balls). Note that the Ge atoms are not positioned at the center between two adjacent $\mathrm{Te}$ atoms but rather shifted to the right. This implies an uncompensated polarization, which is consistent with the known ferroelectric nature of $\alpha$ $\mathrm{GeTe}^{21,25}$. For quantitative analysis, a line profile is also displayed along the red-dashed line in Fig. 1c, which is composed of two kinds of peaks. From the peak positions, the distances of Te(left)-Ge(right) and Ge(left)$\mathrm{Te}$ (right) are estimated to be 2.08 and $1.51 \AA$, respectively [Fig. 1d]. These values are very close to the known values of 2.14 and $1.49 \AA^{26,27}$. Figure 1e shows the selected-area electron diffraction (SAED) pattern of a $5 \mu \mathrm{m}$ spot size, indicating that the GeTe film is highly crystalline with the $R 3 \mathrm{~m}$ space group mixed with a small amount of twin grains, as indicated by the yellow arrow. The ferroelectric nature of GeTe films is also confirmed by a piezoresponse force microscopy (PFM) study (Supplementary Fig. S2), where clear spontaneous polarization is uniformly observed.

Figure $1 \mathrm{f}$ shows the magnetoresistance (MR) of a 50-nmthick GeTe film as a function of the external magnetic field $(B)$ applied perpendicular to the film plane. The MR curves exhibit weak antilocalization (WAL)-like features (sharp dip near $B=0$; for detailed analysis, see Supplementary Note 1), indicating a strong SOC in the film. Using the Fukuyama-Hoshino model for three-dimensional WAL ${ }^{28}$, we fit the MR curves [black dashed lines in Fig. 1f] and obtain a Rashba constant $\left(\alpha_{\mathrm{R}}\right)$ of $\sim 0.3 \times 10^{-10} \mathrm{eV}$ m at $20 \mathrm{~K}$, which is approximately three times larger than that of a strong semiconducting Rashba system ${ }^{2}$.

\section{Harmonic Hall measurements}

An in-plane charge current flowing through an SOC channel induces a perpendicular spin current, which exerts an SOT on the nearby ferromagnet. In this experiment, we extract the magnitude of the SOT in a GeTe $(130 \mathrm{~nm}) / \mathrm{NiFe}$ $(20 \mathrm{~nm})$ structure via harmonic Hall measurements (see the Experimental Section). We investigate a field-like torque $\left(\mathbf{T}_{\mathrm{FL}} \propto \mathbf{m} \times \mathbf{y}\right)$ and a damping-like torque [ $\mathbf{T}_{\mathrm{DL}} \propto \mathbf{m} \times(\mathbf{y} \times$ $\mathbf{m})]$, where $\mathrm{m}$ and $\mathrm{y}$ are unit vectors along the magnetization and perpendicular to both directions of charge-current flow $(x)$ and spin-current flow $(z)$, respectively. Regarding in-plane magnetization, the field-like and damping-like torques correspond to the in-plane $\left(B_{\mathrm{FL}}\right)$ and out-of-plane $\left(B_{\mathrm{DL}}\right)$ effective spin-orbit fields, respectively, as shown in Fig. 2a. We note that according to our coordinate system and geometry, a current-induced Oersted field is in the $-y$ direction for a positive current [Fig. 2a]. The sign of the current-induced Oersted field is also confirmed by the harmonic Hall measurement of a $\mathrm{Cu} / \mathrm{NiFe}$ bilayer where the $\mathrm{Cu}$ layer creates a negligible SOT (Supplementary Fig. S3).

Figure $2 \mathrm{~b}$ shows the optical microscopy image and measurement geometry of the Hall bar structure. An external in-plane magnetic field $B_{\text {ext }}(=25 \mathrm{mT}-4 \mathrm{~T})$ is applied while varying the azimuthal angle $(\varphi)$ between $B_{\text {ext }}$ and the AC current $I$ [Fig. 2a]. Because of the easy-plane anisotropy of the NiFe layer, the magnetization is aligned along $B_{\text {ext }}$. In this field geometry for in-plane magnetization, the first $\left(R_{x y}^{\omega}\right)$ and second $\left(R_{x y}^{2 \omega}\right)$ harmonic Hall resistances are expressed as ${ }^{24,29}$

$$
R_{x y}^{\omega}=R_{\mathrm{PHE}} \sin 2 \varphi,
$$


(a)

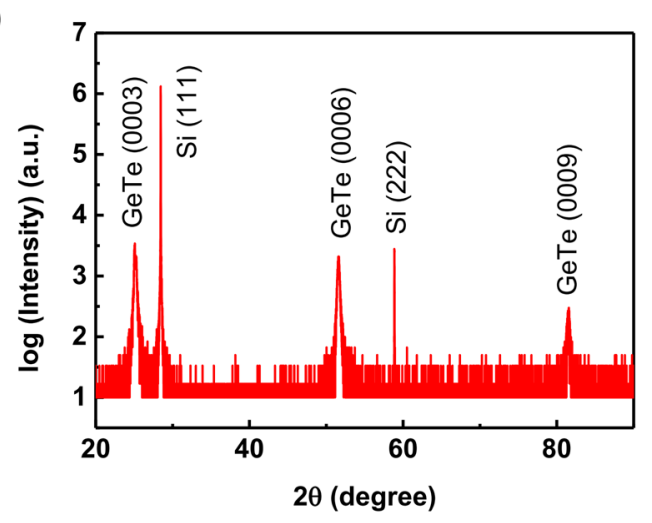

(c)

(e)
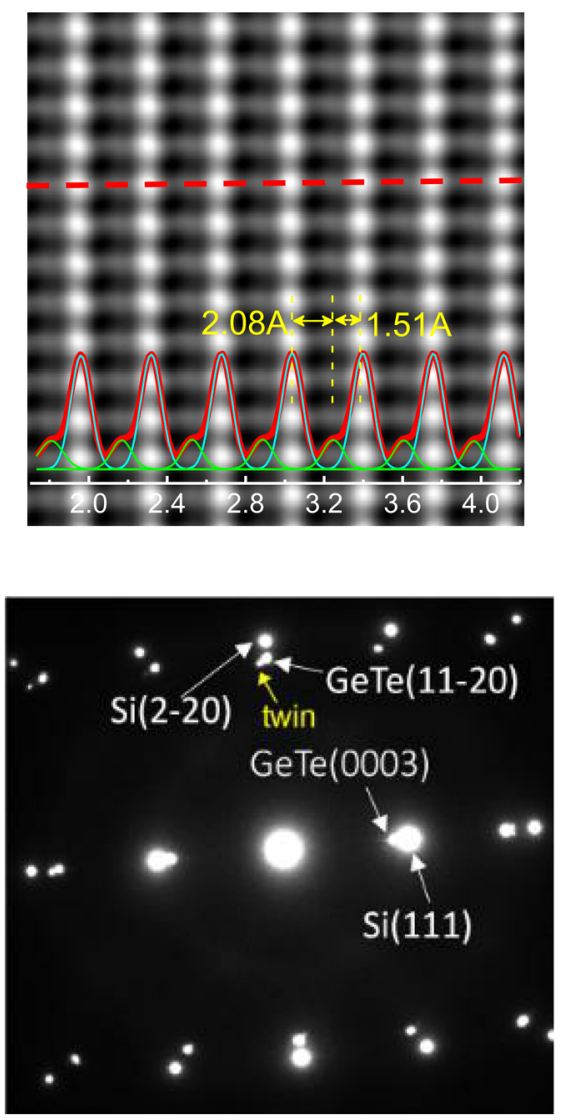

(b)

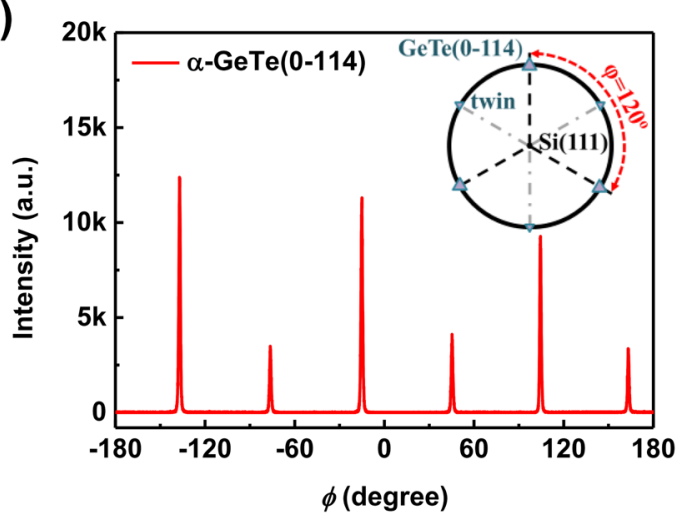

(d)

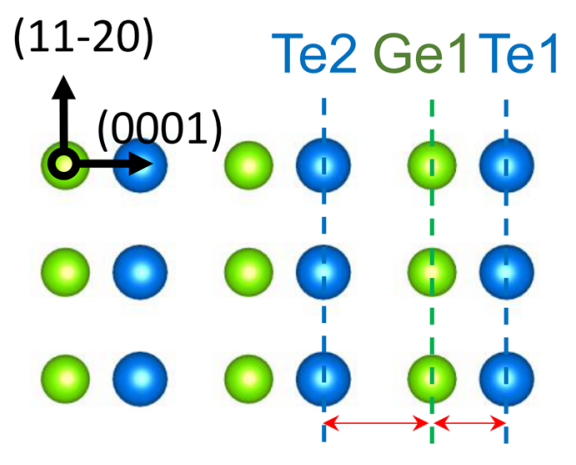

(f)
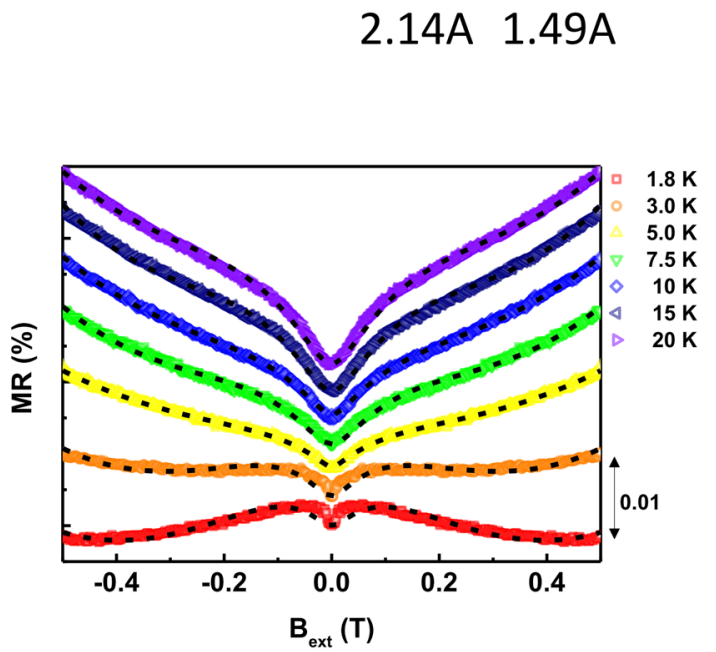

Fig. 1 Structural characteristics and magnetoresistance of epitaxial GeTe thin films. $\mathbf{a}$, $\mathbf{b}$ X-ray diffraction (XRD) of the $2 \theta$ scan (a) and $\phi$-scan around $\mathrm{Si}(111)$ (b), c Fast Fourier transform (FFT)-filtered scanning transmission electron microscopy (STEM) image with a line profile along the reddashed line, where peaks are separated into two representing the positions of the Ge and Te atoms. XRD and TEM analyses were conducted with a 100-nm-thick GeTe film on a Si(111) substrate. d Schematic illustration of the structure of a-GeTe with interplane distances of 1.49 and $2.14 \AA$ for Ge1Te1 and Te2-Ge1, respectively. e Selected-area electron diffraction (SAED) image, f Magnetoresistance (MR) of a 50-nm-thick GeTe film at various temperatures (from 1.8 to $20 \mathrm{~K}$, symbols), together with the fitting curves (black dashed lines) obtained by the Fukuyama-Hoshino model. At $20 \mathrm{~K}$, a Rashba parameter of $a_{R}=0.3 \times 10^{-10} \mathrm{eV}$ is extracted from the magnetoresistance curve.

$$
\begin{aligned}
R_{x y}^{2 \omega}= & 2 R_{\mathrm{PHE}} \frac{B_{\mathrm{FL}}+B_{\mathrm{Oe}}}{B_{\mathrm{ext}}} \cos 2 \varphi \cos \varphi \\
& +\left(R_{\mathrm{AHE}} \frac{B_{\mathrm{DL}}}{B_{\mathrm{eff}}}+A I_{0} \alpha \nabla T+N I_{0} \alpha B_{\mathrm{ext}} \nabla T\right) \cos \varphi \\
= & C_{\mathrm{FL}} \cos 2 \varphi \cos \varphi+C_{\mathrm{DL}} \cos \varphi,
\end{aligned}
$$

where $R_{\mathrm{PHE}}$ is the planar Hall resistance; $R_{\mathrm{AHE}}$ is the anomalous Hall resistance; $B_{\text {eff }}$ is the sum of $B_{\text {ext }}$ and the effective out-of-plane demagnetizing field $B_{\mathrm{d}}$; $A$ is the coefficient of the thermomagnetic effects, including 
(a)

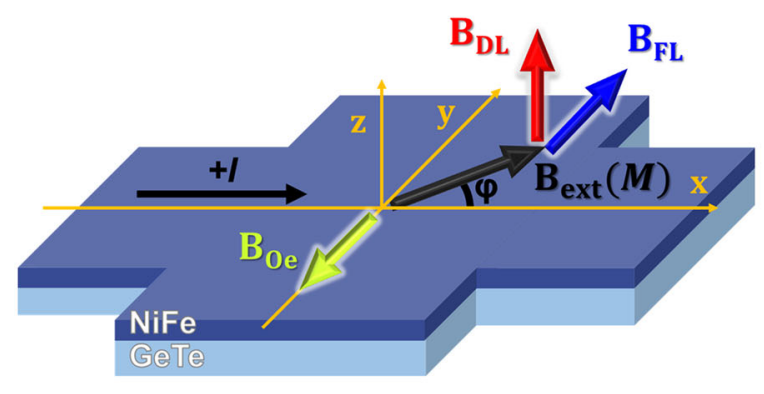

(c)

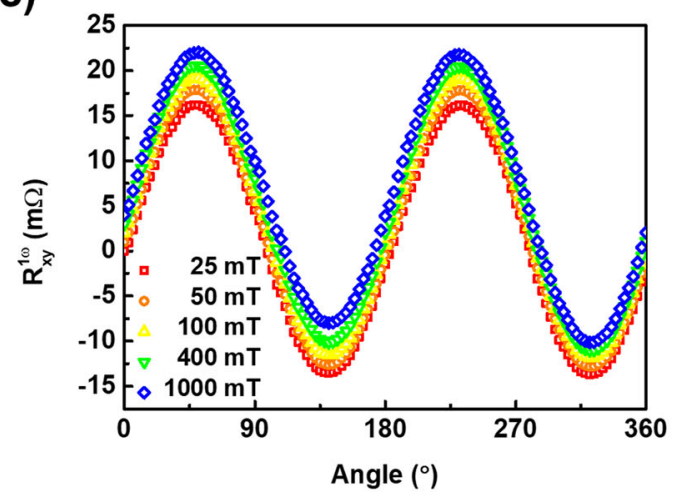

(b)

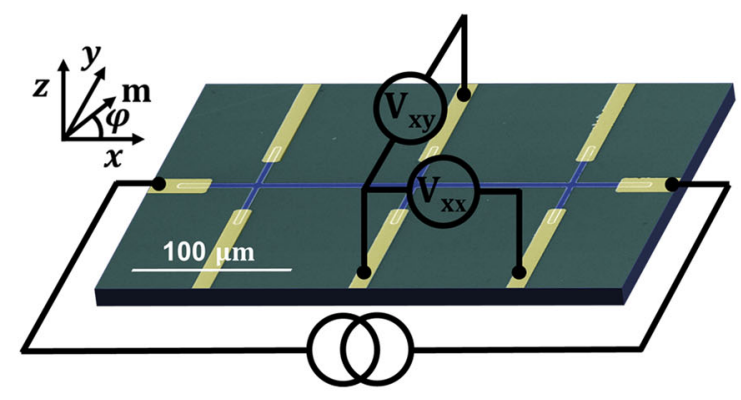

(d)

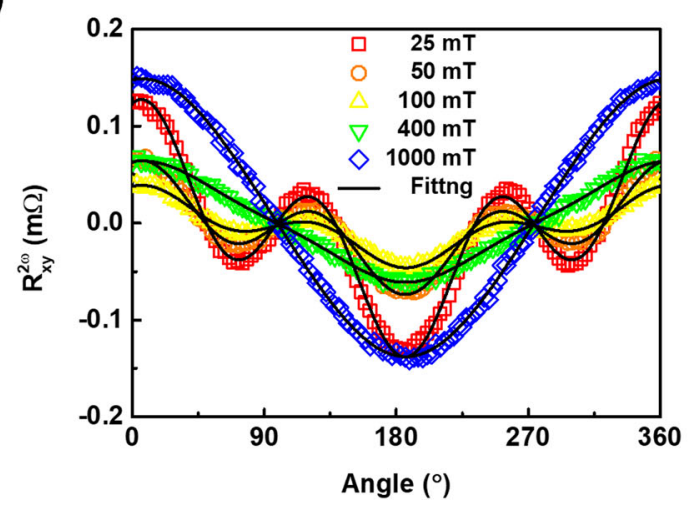

Fig. 2 First and second-harmonic resistances of the GeTe/NiFe bilayer. a Schematic diagram of field-like and damping-like torques. The In-plane $\left(B_{F L}\right)$ and out-of-plane $\left(B_{\mathrm{DL}}\right)$ effective spin-orbit fields correspond to the field-like and damping-like torques, respectively. $\mathbf{b}$ Optical image and measurement geometry of the GeTe/NiFe hybrid device. $\mathbf{c}$, $\mathbf{d}$ Transverse resistances of the first (c) and second-harmonic measurements (d). In $\mathbf{d}$, the experimental data agree with the solid lines, which are the fits obtained from Eq. (2) ( $\left.I_{\mathrm{AC}}=3 \mathrm{~mA}\right)$.

the anomalous Nernst effect and spin-Seebeck effect; $I_{0}$ is the amplitude of the AC current; $\alpha$ is the geometric factor; $\nabla T$ is the vertical temperature gradient; $N$ is the ordinary Nernst coefficient, and $B_{\mathrm{Oe}}$ is the current-induced Oersted field. The vertical temperature gradient may originate from current-induced Joule heating and the asymmetric heat flow between layers. We note that in comparison to ref. ${ }^{24}$, the ordinary Nernst term, which is linear in $B_{\text {ext }}$, is included in Eq. (2) because this term is found to be large in our $\mathrm{GeTe} / \mathrm{NiFe}$ structure, which we show below. A recent experiment ${ }^{29}$ reported that the ordinary Nernst term was also large in a $\mathrm{Bi}-\mathrm{Sb} / \mathrm{Co}$ heterostructure.

Figure 2c, d shows the measured first- and secondharmonic Hall resistances, respectively. The first harmonic Hall resistance $R_{x y}^{\omega}$ follows the angular dependence of $\sin 2 \varphi$ as expected from the planar Hall effect [Eq. (1)]. On the other hand, the second-harmonic Hall resistance $R_{x y}^{2 \omega}$ shows the angular dependences of $\cos \varphi$ and $\cos 2 \varphi \cos \varphi$, which is consistent with Eq. (2) and will be analyzed in detail in the next section.

\section{Field-like torque of the GeTe/NiFe structure}

The field-like torque is described by the in-plane effective spin-orbit field $\left(B_{\mathrm{FL}}\right)$ inherently separated from thermal contributions, as shown in the second-harmonic Hall resistance $\left(R_{x y}^{2 \omega}\right)$ of Eq. (2). Thus, this term can be directly extracted from the first term of Eq. (2). Figure 3a shows the amplitude of the $\cos 2 \varphi \cos \varphi$ term, $C_{\mathrm{FL}}$, in the GeTe $(130 \mathrm{~nm}) / \mathrm{NiFe}(20 \mathrm{~nm})$ bilayer. The $C_{\mathrm{FL}}$ is inversely proportional to the external field $B_{\text {ext }}$, which is consistent with Eq. (2). From the first harmonic results of Fig. 2c and Eq. (1), we find that $R_{\mathrm{PHE}}$ is $14.95 \mathrm{~m} \Omega$. From the channel dimension of the Hall bar structure and resistivities $\left(\rho_{\mathrm{GeTe}}=176.7 \mu \Omega \mathrm{cm}, \rho_{\mathrm{NiFe}}=41.7 \mu \Omega \mathrm{cm}\right)$ along with Ampere's law ${ }^{30}$, we estimate that the current-induced Oersted field $\left(B_{\mathrm{Oe}} / J\right)$ is $-8.1 \mathrm{mT} /\left(10^{7} \mathrm{~A} \mathrm{~cm}^{-2}\right)$, where $J$ is the current density flowing through the GeTe layer. Combining these values with $C_{\mathrm{FL}}=2 R_{\mathrm{PHE}}\left(B_{\mathrm{FL}}+\right.$ $\left.B_{\mathrm{Oe}}\right) / B_{\text {ext }}$, we find a field-like torque $\left(B_{\mathrm{FL}} / /\right)$ of $+12.0 \mathrm{mT} /$ $\left(10^{7} \mathrm{~A} \mathrm{~cm}^{-2}\right)$, which is larger in magnitude than $B_{\mathrm{Oe}} / J$, and, more importantly, its sign is opposite to $B_{\mathrm{Oe}} / J$. This sign difference unambiguously demonstrates that a 


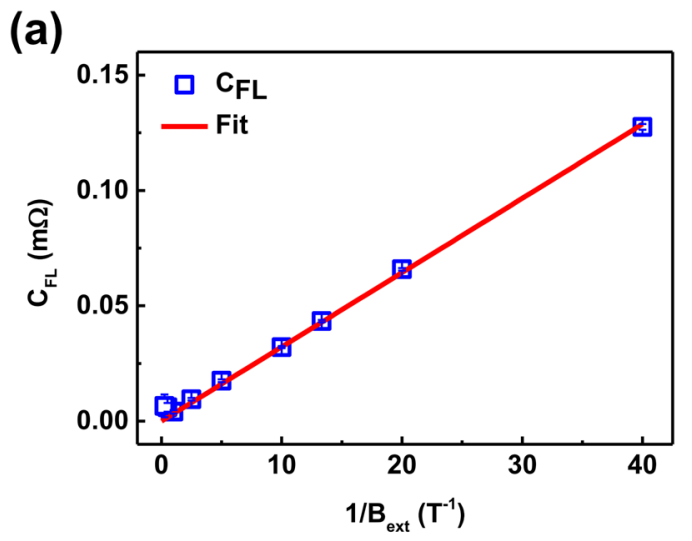

(c)

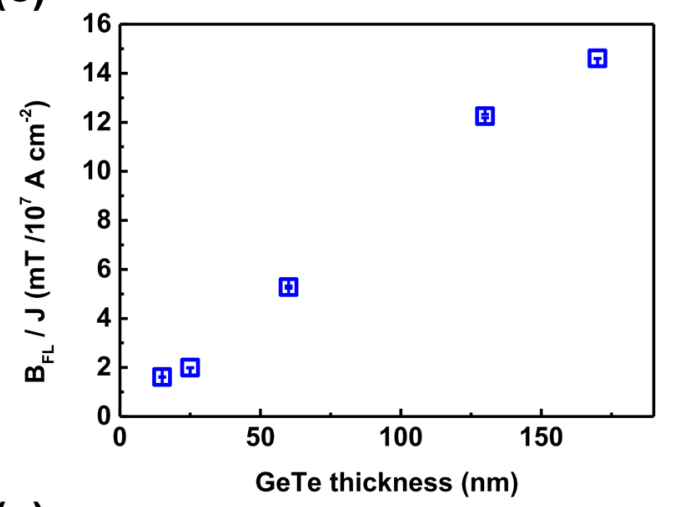

(e)

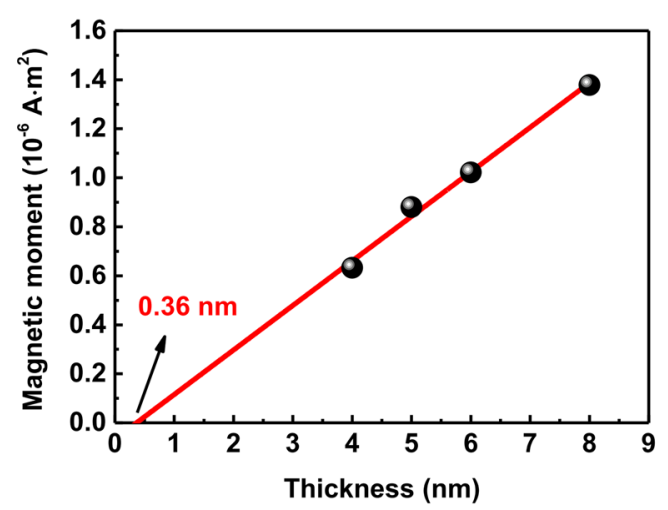

(b)

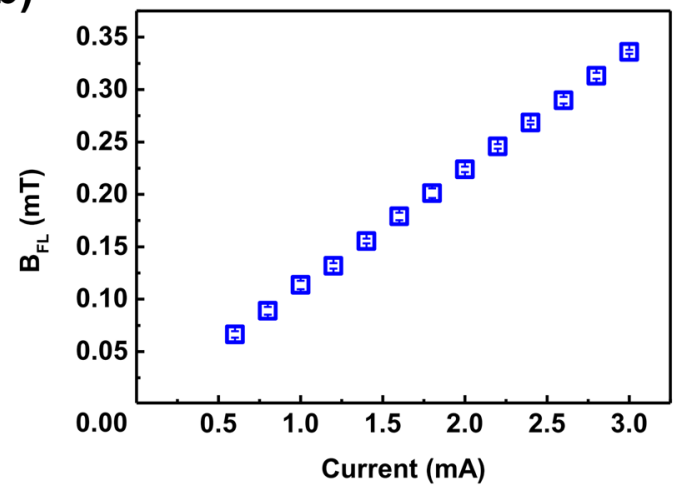

(d)

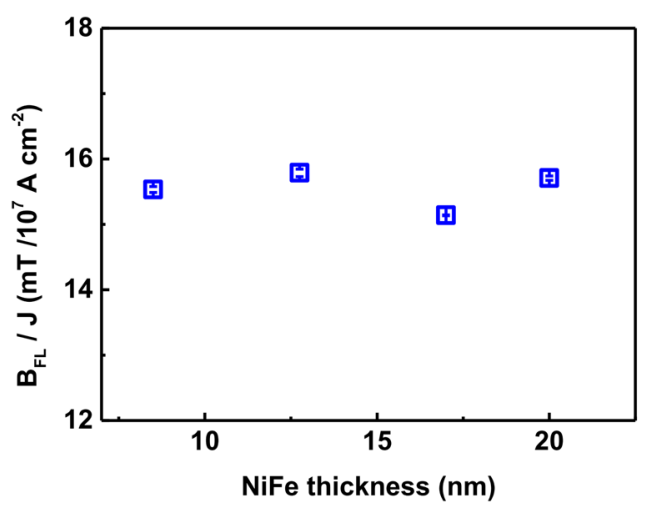

(f)

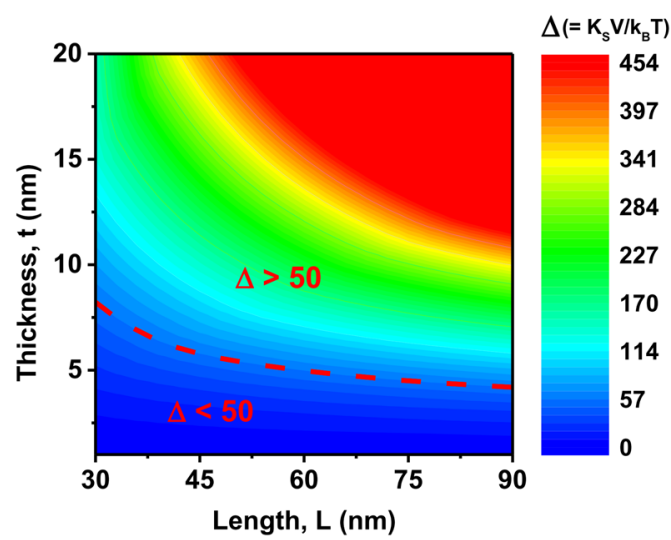

Fig. 3 Field-like torque of the GeTe/NiFe structure. a Amplitude of the field-like term, $C_{\mathrm{FL}}$, as a function of $B_{\mathrm{ext}}^{-1}$. $\mathbf{b}$ Field-like torque, $B_{\mathrm{FL}}$, as a function of the AC current. $\mathbf{c} B_{\mathrm{FL}} / J$ as a function of the GeTe thickness (the NiFe thickness is $20 \mathrm{~nm}$ ). The red curve is the fitting result of Eq. (2). $\mathbf{d} B \mathrm{FL} / J$ as a function of the NiFe thickness (the GeTe thickness is $170 \mathrm{~nm}$ ). e Magnetic moment versus the NiFe thickness deposited on GeTe. The red line is the linear fit, which gives a magnetic dead layer thickness of $0.36 \mathrm{~nm}$. $\mathbf{f}$ Contour of the calculated $\Delta$ as a function of the in-plane length $L$ and the thickness $t$ of the FM layer, assuming an in-plane aspect ratio of 2 and a saturation magnetization of $800 \mathrm{kA} \mathrm{m}^{-1}$. The dotted red curve is the boundary corresponding to $\Delta=50$. In the figures, the error bars representing the standard deviation are smaller than the symbols except where shown.

spin-orbit-originated field-like torque is present in GeTe/ NiFe bilayers. Previous studies reported $B_{\mathrm{FL}}=2 \mathrm{mT} /\left(10^{7}\right.$ $\left.\mathrm{A} \mathrm{cm}^{-2}\right)$ and $4 \mathrm{mT} /\left(10^{7} \mathrm{~A} \mathrm{~cm}^{-2}\right)$ for $\mathrm{Pt} / \mathrm{CoFe}^{31}$ and $\mathrm{Pt} /$ $\mathrm{Co}^{32}$ channels, respectively. In a $\mathrm{Ta} / \mathrm{CoFeB}$ channel $^{33}$, the observed spin torques were $B_{\mathrm{FL}}=-2.1 \mathrm{mT} /\left(10^{7} \mathrm{Acm}^{-2}\right)$. Thus, the $B_{\mathrm{FL}}$ in our system is as large of a value as that obtained in the heavy metal system. The field-like torque is found to be linear with the AC current [Fig. 3b; see 
Supplementary Fig. S4 for the detailed harmonic curves], validating the estimation of $B_{\mathrm{FL}} / J$. We also confirm that the $\cos 2 \varphi \cos \varphi$ term is absent in a $\mathrm{GeTe} / \mathrm{Cu}$ structure (Supplementary Fig. S5), showing that $B_{\mathrm{FL}}$ is not an artifact originating from $\mathrm{GeTe}$ itself.

Assuming that the bulk SOC of GeTe is the only source of spin current, one can analyze the magnitude of $B_{\mathrm{FL}} / J$ based on the spin drift-diffusion model with the quantum mechanical boundary condition ${ }^{8}$. This model predicts that $B_{\mathrm{FL}} / J$ increases and then saturates when the NM thickness exceeds its spin diffusion length. It also predicts that $B_{\mathrm{FL}} / J$ is inversely proportional to the FM thickness, as reported in a recent experiment ${ }^{34}$. To check whether this diffusion model is applicable to our study, we performed harmonic Hall measurements on $\mathrm{GeTe} / \mathrm{NiFe}$ bilayers with varying GeTe thicknesses [Fig. 3c] and NiFe thicknesses [Fig. 3(d)]. Regarding the GeTe thickness dependence [Fig. 3c], $B_{\mathrm{FL}} / J$ shows a slightly nonlinear dependence on the GeTe thickness but does not exhibit a clear saturation behavior. One possible explanation is that the spin diffusion length of GeTe is comparable to or larger than the largest tested GeTe thickness $(170 \mathrm{~nm})$. However, this explanation is unlikely because the spin diffusion length of most spin-orbit channels is on the nanometer scale ${ }^{35}$. Moreover, for the NiFe-thickness dependence [Fig. 3d], we find that $B_{\mathrm{FL}} / J$ is almost constant even with a threefold change in the NiFe thickness. This behavior is evidently inconsistent with the prediction of the spin drift-diffusion model. Haney et al. ${ }^{8}$ reported Boltzmann solutions for the SOT but did not consider the bulk Rashba SOC in the normal metal layer. Another missing part is that it does not consider the SOC in ferromagnets. A recent theory ${ }^{36}$ based on quantum Boltzmann equations reported that the SOC in a ferromagnet also generates an SOT. In this respect, we need a theory based on the Boltzmann equations, which takes into account the $\mathrm{SOC}$ in ferromagnets and the bulk Rashba SOC in the nonmagnetic layer, which demands further theoretical progress. Recently, nonreciprocal charge transport of the GeTe channel was observed ${ }^{37}$, and this term is possibly added to our observed SOT signal. Moreover, this unique property of the field-like torque, which maintains a large magnitude even for a thick FM layer, is applicable for scalable in-plane SOT memories [Fig. 3f, see the "Discussion" section].

We check several possibilities for the unconventional thickness dependence of field-like torque. We first check whether a thick magnetic dead layer is formed at the $\mathrm{GeTe} / \mathrm{NiFe}$ interface. We find that the magnetic dead layer is only $0.36 \mathrm{~nm}$ thick [Fig. 3e], so we exclude this possibility for the unconventional dependence on NiFe thickness of field-like torque. Recent studies have reported that oxidation significantly affects the $\mathrm{SOT}^{38-40}$, we also checked whether an oxide layer is formed at the
GeTe/NiFe interface with energy dispersive spectroscopy (EDS) but found no noticeable oxidation signature (Supplementary Fig. S6).

However, another possibility is the interface SOC at the $\mathrm{GeTe} / \mathrm{NiFe}$ interface. The spin drift-diffusion model is obtained by integrating the Boltzmann equation; thus, the Boltzmann equation is better suited to describe in-plane transport ${ }^{8}$. The Boltzmann transport calculation ${ }^{8}$ for the interfacial SOC shows that the scaled field-like torque [= (field-like torque) $\times$ (FM thickness)] increases and then saturates with increasing FM thickness. In other words, it predicts that the field-like torque is not scaled with 1 /(FM thickness) but is more or less constant in some ranges of FM thickness. Our observation [Fig. 3d] is qualitatively consistent with this prediction that considers the interface SOC. Therefore, we attribute the unconventional NiFethickness dependence of field-like torque to the interface effect, which may be enhanced due to the bulk Rashba SOC of GeTe.

\section{Damping-like torque of the GeTe/NiFe structure}

Based on the second-harmonic Hall resistance $\left(R_{x y}^{2 \omega}\right)$, we attempted to extract the magnitude of damping-like torque in the GeTe/NiFe bilayers. The damping-like torque is expressed as the out-of-plane effective spin-orbit field $\left(B_{\mathrm{DL}}\right)$, which can be extracted from the amplitude of the $\cos \varphi$ term in Eq. (2). However, several thermomagnetic effects $^{24,29}$, such as the ordinary Nernst effect, the anomalous Nernst effect, and the spin-Seebeck effect, are also present in the second-harmonic signals. The damping-like term amplitude $C_{\mathrm{DL}}=R_{\mathrm{AHE}} \frac{B_{\mathrm{DL}}}{B_{\mathrm{ext}}+B_{\mathrm{d}}}+$ $A I_{0} \alpha \nabla T+N I_{0} \alpha B_{\text {ext }} \nabla T$ includes three terms that depend on $B_{\text {ext }}$ in different ways. From the independent experiments, we find an $R_{\mathrm{AHE}}$ of $3.41 \mathrm{~m} \Omega$ and a $B_{\mathrm{d}}$ of $1 \mathrm{~T}$ (Supplementary Fig. S7). As shown in Fig. $4 \mathrm{a}, C_{\mathrm{DL}}$ fits well to Eq. (2). However, the third term of $C_{\mathrm{DL}}, N I_{0} \alpha B_{\text {ext }} \nabla T$, which is linear in $B_{\text {ext, }}$ dominates the other two terms [Fig. $4 \mathrm{~b}]$. Even though the first $\left(R_{\mathrm{AHE}} B_{\mathrm{DL}} / B_{\text {eff }}\right)$ and second ( $\left.A I_{0} \alpha \nabla T\right)$ terms have different dependences on $B_{\text {ext }}$, the combined magnitude of these terms is too small to reliably separate. As a result, the damping-like torque of the $\mathrm{GeTe} / \mathrm{NiFe}$ bilayer is believed to be much smaller than the field-like torque. From the spin-torque ferromagnetic resonance measurement, we also confirm the weak damping-like torque (Supplementary Note 2 and Supplementary Fig. S8).

\section{Discussion}

Now, we focus on the field-like torque and its possible utilization. An interesting observation in our work is that the field-like torque is weakly dependent on the FM thickness, which is in stark contrast to the typical inverse proportionality of the SOT magnitude to the FM 
(a)

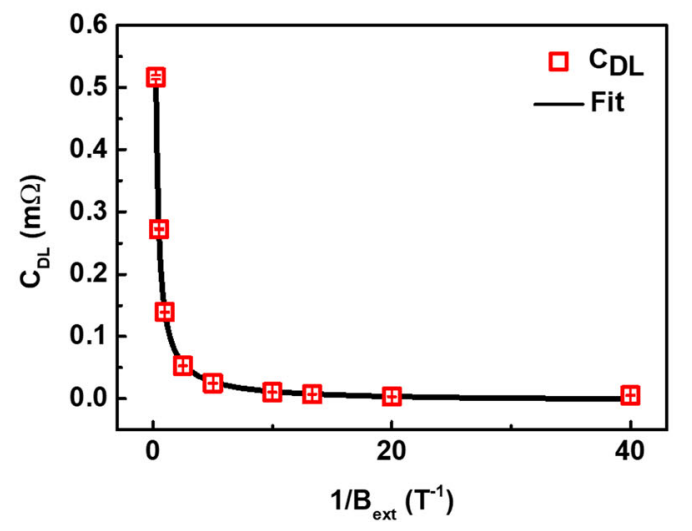

(b)

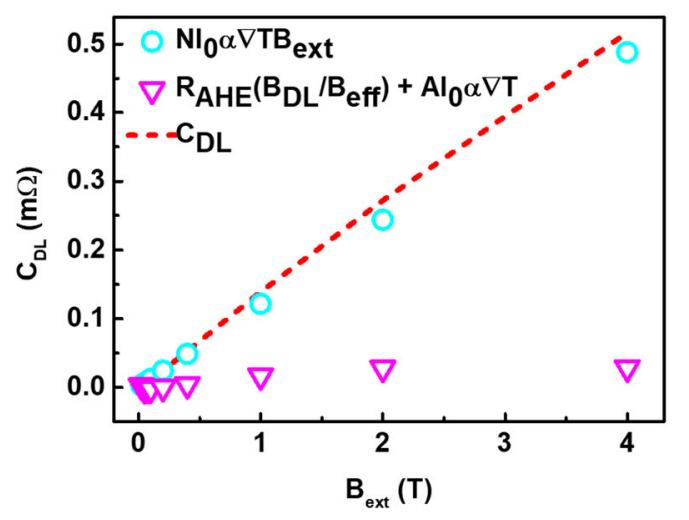

Fig. 4 Damping-like torque of the GeTe/NiFe structure. a Amplitude of the damping-like term, $C_{\mathrm{DL}}$, as a function of $B_{\text {ext }}^{-1}$. b Comparison between the ordinary Nernst term and other terms. The ordinary Nernst term is proportional to $B_{\text {ext }}$ and dominates the other two terms.

thickness. This suggests that the bulk SOC of GeTe is not the only source of spin current in the GeTe/NiFe bilayers. Rather, the spin current is noticeably attributed to the interfacial SOC at the GeTe/NiFe interface. The large field-like torque indicates a large interfacial SOC effect. The bulk Rashba effect of GeTe itself may have a role in the large interfacial SOC effect, which demands further theoretical studies.

Another interesting observation is that the field-like torque is large even with a thick FM layer. When considering the FM thickness, the field-like torque of the $\mathrm{GeTe} / \mathrm{NiFe}$ bilayer in this study is noticeably large, and this large field-like torque suggests that the GeTe/FM bilayers can be used for scalable in-plane SOT magnetic random access memories (MRAMs). Even though the SOT is able to switch the in-plane magnetization ${ }^{12,41}$, inplane MRAMs suffer from inferior scalability to perpendicular MRAMs. The thermal energy barrier $\Delta$ of in-plane MRAM is given by $K_{\mathrm{s}} V / k_{\mathrm{B}} T$, where $K_{\mathrm{s}}$ is the shape anisotropy, $V$ is the FM-layer volume, and $k_{\mathrm{B}} T$ is the thermal energy at room temperature. Figure $3 \mathrm{f}$ shows a contour map of the calculated $\Delta$ as a function of the in-plane length $L$ and thickness $t$ of the FM layer, assuming an inplane aspect ratio of 2 and a saturation magnetization of $800 \mathrm{kA} / \mathrm{m}$. It clearly shows that $\Delta>50$ for 10 -year retention cannot be reached for a thin FM layer. One has to increase the FM thickness to meet the criteria of $\Delta>50$. However, when the SOT is inversely proportional to the FM thickness, it costs an increased write current, resulting in high power consumption. In this respect, the large field-like torque of the $\mathrm{GeTe} / \mathrm{NiFe}$ bilayers for a thick NiFe layer and its weak dependence on the NiFe thickness are attractive for scalable in-plane MRAMs.

Finally, we checked the possibility of electrically controlling the SOT of GeTe/NiFe bilayers. This is one of our original motivations of this study because GeTe is a wellknown ferroelectric material and is expected to exhibit gate-controlled electric polarization and subsequent Rashba effect modulation in a nonvolatile manner. However, we find no noticeable modulation of the fieldlike torque by gating (Supplementary Fig. S9) due to the relatively high conductivity of $\mathrm{GeTe}$ and/or the inefficiency of back-gating. Therefore, we need to engineer the material itself or to develop an alternative method for electrically controlling the polarization, which is beyond the scope of this work.

\section{Materials and methods}

\section{Material growth and characterization}

$\alpha$-GeTe thin films have been grown on $\mathrm{Si}(111)$ substrates by the thermal evaporation of single elemental sources of $\mathrm{Ge}$ and $\mathrm{Te}$. Just before loading into the growth chamber, the substrate was carefully prepared via three cleaning steps: (1) in piranha solution $\left(\mathrm{H}_{2} \mathrm{O}_{2}: \mathrm{H}_{2} \mathrm{SO}_{4}=\right.$ 1:3) for 10 min, (2) in SC-1 solution $\left(\mathrm{NH}_{3}: \mathrm{H}_{2} \mathrm{O}_{2}: \mathrm{H}_{2} \mathrm{O}=\right.$ 1:4:20) for $10 \mathrm{~min}$, and (3) in diluted HF solution for a few minutes to remove the native oxide. After loading the substrate, the growth chamber was evacuated below $5 \times$ $10^{-8}$ Torr, and the substrate was quickly heated and held at $270{ }^{\circ} \mathrm{C}$ for $1 \mathrm{~h}$. The fluxes of $\mathrm{Ge}$ and $\mathrm{Te}$ gas were separately controlled by using a thickness monitor and optimized with respect to the composition, crystallinity, and surface morphology. After the optimized flux of Ge and Te gas was stabilized, the growth started with the substrate temperature at $270{ }^{\circ} \mathrm{C}$, and the thickness of GeTe was controlled by the deposition time with an average deposition rate of $0.44 \AA \mathrm{s}^{-1}$. The structural characteristics of the grown GeTe films were investigated by using X-ray diffraction (ATX-G, Rigaku), atomic force microscopy (AFM, XE-70, Park systems), and 
transmission electron microscopy (TEM, TitanTM 80-300, FEI). The composition of the GeTe films was analyzed by using Auger electron spectroscopy (AES, PHI-700, ULVAC-PHI).

\section{Device fabrication}

The device consists of a $\mathrm{GeTe} / \mathrm{Ni}_{81} \mathrm{Fe}_{19} / \mathrm{MgO} / \mathrm{Ta}$ multilayer, where a $\mathrm{MgO}(2 \mathrm{~nm}) / \mathrm{Ta}(2 \mathrm{~nm})$ layer is deposited to protect the ferromagnet layer. After the grown GeTe films underwent an ion-milling process, the ferromagnetic layer and the capping layers were deposited by DC magnetron sputtering without vacuum breaking (see Supplementary Fig. S10 for the FM characteristics). The transport channels were patterned by photolithography and Ar-ion milling. Then, contact electrodes made of $\mathrm{Ti}$ $(5 \mathrm{~nm}) / \mathrm{Au}(100 \mathrm{~nm})$ were formed by photolithography and lift-off. The lateral dimension of the channel was $100 \mu \mathrm{m} \times 6 \mu \mathrm{m}$.

\section{Harmonic measurement}

All measurements were performed in a physical property measurement system (PPMS) at room temperature. The Hall voltage was measured by supplying AC current from a Keithley 6221 device and simultaneously monitoring the first- and second-harmonic Hall voltages by SR850 and SR860 lock-in amplifiers, respectively. All harmonic transport measurements were performed at room temperature with an alternating current at a frequency of $17 \mathrm{~Hz}$.

\section{Acknowledgements}

This work was mainly supported by KIST Institutional Programs (2E30600, 2E30610, 2V05750). H.C.K. and O.J.L. acknowledge the support from the National Research and Development Program through the National Research Foundation of Korea (NRF), which is funded by the Ministry of Science and ICT (2019M3F3A1A02071509, 2020M3F3A2A01081635), and the KU-KIST Institutional Program. S.W.C., J.K., and S.L. acknowledge the support from the National Research Foundation Program (NRF-2019M3F3A1A02072175). K.-J.L. acknowledges the support from the the National Research Foundation of Korea (NRF) (NRF-2020M3F3A2A01082591).

\section{Author details}

${ }^{1}$ Center for Spintronics, Korea Institute of Science and Technology, Seoul, Korea. ${ }^{2}$ Department of Applied Physics, Korea University, Sejong, Korea. ${ }^{3}$ Center for Neuromorphic Engineering, Korea Institute of Science and Technology, Seoul, Korea. ${ }^{4}$ Department of Materials Science and Engineering, Seoul National University, Seoul, Korea. ${ }^{5}$ School of Advanced Materials and Engineering, Sungkyunkwan University (SKKU), Suwon, Korea. ${ }^{6}$ Division of Nano \& Information Technology, Korea University of Science and Technology, Daejon, Korea. ${ }^{7}$ KU-KIST Graduate School of Converging Science and Technology, Korea University, Seoul, Korea. ${ }^{8}$ Department of Materials Science and Engineering, Korea University, Seoul, Korea. ${ }^{9}$ Department of Physics, Korea Advanced Institute of Science and Technology, Daejeon, Korea

\section{Author contributions}

J.J. and S.W.C. contributed equally to the work. H.C.K. and S.L. planned the experiment and supervised the research. J.J. and S.W.C. fabricated the devices and collected the data. O.L., J.H., J.Y.P., and S.H. contributed important ideas for sample fabrication. S.J. and Y.K. obtained the PFM images. H.-W.K. and K.-J.L. performed the numerical calculations. H.C.K., S.L., and K.-J.L. analyzed the data and wrote the manuscript with help from all co-authors.

Conflict of interest

The authors declare no competing interests.

\section{Publisher's note}

Springer Nature remains neutral with regard to jurisdictional claims in published maps and institutional affiliations.

Supplementary information The online version contains supplementary material available at https://doi.org/10.1038/s41427-021-00344-6.

Received: 8 July 2020 Revised: 18 October 2021 Accepted: 21 October 2021.

Published online: 26 November 2021

\section{References}

1. Datta, S. \& Das, B. Electronic analog of the electro-optic modulator. Appl. Phys. Lett. 56, 665-667 (1990).

2. Koo, H. C. et al. Control of spin precession in a spin-injected field effect transistor. Science 325, 1515-1518 (2009).

3. Wunderlich, J. et al. Spin Hall effect transistor. Science 330, 1801-1804 (2010).

4. Sasaki, A. et al. Direct determination of spin-orbit interaction coefficients and realization of the persistent spin helix symmetry. Nat. Nanotechnol. 9, 703-709 (2014).

5. Choi, W. Y. et al. Electrical detection of coherent spin precession using the ballistic intrinsic spin Hall effect. Nat. Nanotechnol. 10, 666-670 (2015).

6. Manchon, A., Koo, H. C., Nitta, J., Frolov, S. M. \& Duine, R. A. New perspectives for Rashba spin-orbit coupling. Nat. Mater. 14, 871-882 (2015).

7. Manchon, A. \& Zhang, S. Theory of nonequilibrium intrinsic spin torque in a single nanomagnet. Phys. Rev. B 78, 212405 (2008).

8. Haney, P. M., Lee, H.-W., Lee, K.-J., Manchon, A. \& Stiles, M. D. Current induced torques and interfacial spin-orbit coupling: Semiclassical modeling. Phys. Rev. B 87, 174411 (2013)

9. Wang, L. et al. Giant room temperature interface spin Hall and inverse spin Hall effects. Phys. Rev. Lett. 116, 196602 (2016).

10. Amin, V. P., Zemen, J. \& Stiles, M. D. Interface-generated spin currents. Phys. Rev. Lett. 121, 136805 (2018).

11. Miron, I. M. et al. Perpendicular switching of a single ferromagnetic layer induced by in-plane current injection. Nature 476, 189-193 (2011).

12. Liu, L. et al. Spin-torque switching with the giant spin Hall effect of tantalum. Science 336, 555-558 (2012).

13. Lee, S.-W. \& Lee, K.-J. Emerging three-terminal magnetic memory devices. Proc IEEE 104, 1831-1843 (2016).

14. Chen, Y. L. et al. Discovery of a single topological Dirac fermion in the strong inversion asymmetric compound BiTeCl. Nat. Phys. 9, 704-708 (2013).

15. Ishizaka, K. et al. Giant Rashba-type spin splitting in bulk BiTel. Nat. Mater. 10 521-526 (2011)

16. Sante, D. D., Barone, P., Bertacco, R. \& Picozzi, S. Electric control of the giant Rashba effect in bulk GeTe. Adv. Mater. 25, 509-513 (2013).

17. Picozzi, S. Ferroelectric Rashba semiconductors as a novel class of multifunctional materials. Front. Phys. 2, 10 (2014)

18. Narayan, A. Class of Rashba ferroelectrics in hexagonal semiconductors. Phys. Rev. B 92, 220101 (2015).

19. Chattopadhyay, T., Boucherle, J. X. \& vonSchnering, H. G. Neutron diffraction study on the structural phase transition in GeTe. J. Phys. C Solid State Phys. 20, 1431-1440 (1987).

20. Polking, M. J. et al. Ferroelectric order in individual nanometre-scale crystals. Nat. Mater. 11, 700-709 (2012).

21. Krempaský, J. et al. Disentangling bulk and surface Rashba effects in ferroelectric a -GeTe. Phys. Rev. B 94, 205111 (2016).

22. Wenxu Zhang et al. Tuning spin Hall conductivity in GeTe by ferroelectric polarization. Phys. Status Solidi B 257, 2000143 (2020).

23. Wang, $H$. et al. Spin Hall effect in prototype Rashba ferroelectrics GeTe and SnTe. Npj Comput. Mater. 6, 1-7 (2020).

24. Avci, C. O. et al. Interplay of spin-orbit torque and thermoelectric effects in ferromagnet/normal-metal bilayers. Phys. Rev. B 90, 224427 (2014). 
25. Rinaldi, C. et al. Ferroelectric control of the spin texture in GeTe. Nano Lett. 18, 2751-2758 (2018).

26. Wang, R. et al. Toward truly single crystalline GeTe films: the relevance of the substrate surface. J. Phys. Chem. C 118, 29724-29730 (2014)

27. Wuttig, M. et al. The role of vacancies and local distortions in the design of new phase-change materials. Nat. Mater. 6, 122-128 (2007)

28. Fukuyama, H. \& Hoshino, K. Effect of spin-orbit interaction on magnetoresistance in the weakly localized regime of three-dimensional disordered systems. J. Phys. Soc. Jpn 50, 2131-2132 (1981).

29. Roschewsky, N. et al. Spin-orbit torque and Nernst effect in Bi-Sb/Co heterostructures. Phys. Rev. B 99, 195103 (2019).

30. Liu, L., Moriyama, T., Ralph, D. C. \& Buhrman, R. A. Spin-torque ferromagnetic resonance induced by the spin Hall effect. Phys. Rev. Lett. 106, 036601 (2011).

31. Emori, S. et al. Current-driven dynamics of chiral ferromagnetic domain walls. Nat. Mater. 12, 611-616 (2013)

32. Garello, K. et al. Symmetry and magnitude of spin-orbit torques in ferromagnetic heterostructures. Nat. Nanotechnol. 8, 587-593 (2013).

33. Avci, C. O. et al. Fieldlike and antidamping spin-orbit torques in as-grown and annealed Ta/CoFeB/MgO layers. Phys. Rev. B 89, 214419 (2014).
34. $\mathrm{Yu}$, J. et al. Long spin coherence length and bulk-like spin-orbit torque in ferrimagnetic multilayers. Nat. Mater. 18, 29-34 (2019).

35. Manchon, A. et al. Current-induced spin-orbit torques in ferromagnetic and antiferromagnetic systems. Rev. Mod. Phys. 91, 035004 (2019).

36. Kim, K. W. \& Lee, K. J. Generalized spin drift-diffusion formalism in the presence of spin-orbit interaction of ferromagnets. Phys. Rev. Lett. 125 207205 (2020).

37. Li, Y. et al. Nonreciprocal charge transport up to room temperature in bulk Rashba semiconductor a-GeTe. Nat. Commun. 12, 540 (2021).

38. Qiu, X. et al. Spin-orbit-torque engineering via oxygen manipulation. Nat. Nanotechnol. 10, 333-338 (2015).

39. Demasius, K.-U. et al. Enhanced spin-orbit torques by oxygen incorporation in tungsten films. Nat. Commun. 7, 10644 (2016)

40. An, H., Kageyama, Y., Kanno, Y., Enishi, N. \& Ando, K. Spin-torque generator engineered by natural oxidation of Cu. Nat. Commun. 7, 13069 (2016).

41. Fukami, S., Anekawa, T., Zhang, C. \& Ohno, H. A spin-orbit torque switching scheme with collinear magnetic easy axis and current configuration. Nat. Nanotechnol. 11, 621-625 (2016). 